\title{
The neutrophil-to-lymphocyte ratio on admission is a good predictor for all-cause mortality in hypertensive patients over 80 years of age
}

Xiaonan Sun ${ }^{1+} \mathbb{D}$, Leiming Luo ${ }^{1 *+}$, Xiaoqian Zhao ${ }^{2}$, Ping Ye ${ }^{1}$ and Ruixue Du ${ }^{1}$

\begin{abstract}
Background: Immuno-inflammation plays a major role in the process of hypertension. We aimed to evaluate the association between inflammatory markers, neutrophil-to-lymphocyte ratio (NLR), red cell distribution width (RDW) and all-cause mortality in elderly patients with hypertension.

Methods: A total of 341 hypertensive patients over 80 years of age were included to this study. The NLR and RDW were measured on admission and all the selected patients were followed up for up to 90 days. Kaplan-Meier curves were plotted to evaluate the association between the NLR and the all-cause mortality at follow-up. Using Cox regression models, we investigated the prognostic value of NLR and RDW for all-cause mortality.

Results: Patients with higher quartile of NLR linked to high mortality in hypertensive patients at 90 day after admission $\left(16.47 \%, 13.25 \%, 1.14 \%, 1.17 \%\right.$ respectively; $\left.X^{2}=20.581, P=0.000\right)$. Surviving patients had lower RDW $(13.61 \pm 1.37$ VS $14.18 \pm 1.38, p=0.041)$ and NLR $(4.97 \pm 5.72$ VS $7.95 \pm 6.88, p=0.011)$. The receiver operating curve (ROC) of the NLR for all-cause mortality had an area under the curve (AUC) $=0.714$ (95\%Cl: 0.629-0.798, $P=0$. 000), with acritical value of 2.97 , with sensitivity of $92.6 \%$, and a specificity of $52.5 \%$. The ROC of the RDW to predict all-cause mortality, had an AUC =0.654 (95\%Cl:0.548-0.761, $P=0.008$ ), with acritical value of 13.2\%.The Kaplan-Meier curve showed a significant difference between different NLR levels $(p=0.002)$. Multivariate Cox proportional hazard analysis shown 3rd quartile of $\mathrm{NLR}(\mathrm{RR}=9.646,95 \% \mathrm{Cl} 1.302-34.457, P=0.041)$ and 4th quartiles $(\mathrm{RR}=16.451,95 \% \mathrm{Cl} 2$. $137-66.643, P=0.007$ ) were found to independently predict all-cause death in hypertensive patients over 80 years of age. Higher rank of NLR was link to higher incidence of all-cause death for such patients.
\end{abstract}

Conclusion: The findings of the present study demonstrate the potential utility of NLR in risk stratification of elderly patients with hypertension to provide information for clinical treatment strategies.

Keywords: Neutrophil-to-lymphocyte ratio, Red blood cell distribution, Hypertension, All-cause mortality

\section{What is already known about this topic?}

NLR and RDW have been proved to be a good indicator of the prognosis of a variety of diseases, such as cancer, coronary heart disease and pulmonary embolism.

\section{What does this article add?}

Higher NLR levels in hypertensive patients aged over 80 admitted to the hospital are good predictors for allcause mortality 90 days after admission.

\section{Background}

The role of inflammatory and oxidative stress in cardiovascular diseases has been extensively investigated in multiple studies. Many of them have shown a strong and consistent relationship between cardiovascular diseases and inflammation markers, such as, C-reactive protein

* Correspondence: Lleim@sina.com

${ }^{\dagger}$ Equal contributors

${ }^{1}$ Department of Geriatric Cardiology, Chinese People's Liberation Army General Hospital, 28 Fuxing Road, Beijing 100853, People's Republic of China Full list of author information is available at the end of the article 
(CRP) [1]. Both the neutrophil to lymphocyte ratio (NLR) and the red blood cell distribution (RDW) are novel, inexpensive and easily accessible inflammatory markers which have been shown to be associated with various cardiovascular diseases [2, 3].

The NLR is a ratio of two different yet complementary immune pathways, which serves as a marker of poor general health and physiological stress. Indeed, it has been proved to be a good indicator of the prognosis of a variety of diseases, especially in patients with systemic lupus erythematous (SLE) [4] and cancer [5]. Regarding the RDW, the considerable available evidence suggests that the clinical use of the RDW may be broadened beyond the conventional boundaries of erythrocyte disorders, in particular for assisting the diagnosis and prognosis of patients with acute coronary syndrome (ACS), ischemic cerebrovascular disease, peripheral artery disease (PAD), heart failure (HF) and atrial fibrillation $(\mathrm{AF})[6]$.

Hypertension is one of the most common cardiovascular diseases in the elderly patients. Inflammation and oxidative stress have been implicated in the pathogenesis of hypertension and are the hotspot of hypertension research lately. Patients over 80 years of age are a special group population, usually coming with coronary heart disease, diabetes mellitus (DM), hyperlipidemia, which means that a variety of risk factors superposition together. It was found out there is a certain difference existed between old patients who are aged above 80 and ordinary senile patients indeed through our clinical experience. Such very elderly patients showed differences in the clinical manifestations, treatment response and prognosis and needed more attention. For such kind of patients admitted for all kinds of reasons, whether inflammation markers, such as NLR as well as RDW, can be a predictor for all-cause mortality is still not clear. Accordingly, we designed this preliminary study to investigate the role of these inflammation makers in the evaluation of the prognosis of hypertensive patients.

The aim of this study was to identify independent inflammation predictors of all-cause mortality in hospitalized elderly hypertensive patients, so that such high-risk patients can be identified with sufficient diagnostic accuracy to justify close monitoring or even initiation of secondary prevention hypertension.

\section{Methods}

The current study was conducted at Chinese People's Liberation Army general hospitals with full ethical approval of the Human Investigation Committee in 2010. In addition, informed consent was obtained from all patients.

This study included patients with hypertension who were diagnosed using the criteria listed in Chinese
Hypertension Prevention Guide (2010) [7], hospitalized from January 2011 to December 2013, and aged $>80 \mathrm{y}$. These patients were identified based on previous medical history. Patients with malignant tumors were excluded from the study.

Data on demographic characteristics, such as age, sex, lifestyle (smoking, drinking) and basic medical history, were based on a questionnaire survey of the patients, as well as past medical records. The data collected included history of coronary artery disease (CAD), diabetic mellitus (DM), chronic heart failure, dyslipidemia, chronic kidney disease and anemia. General health parameters, such as height, weight, which were used to calculate body mass index, systolic blood pressure and diastolic blood pressure, resting heart rate, respiratory rate were determined inenrolled patients.

Routine blood tests, including leukocyte, red blood cells, neutrophils $(\mathrm{N})$, lymphocyte $(\mathrm{L})$, red blood cell distribution (RDW), hemoglobin (Hgb), platelet, platelet distribution (PDW) were performed on admission for all the patients. In addition, clinical tests of blood biochemistry, liver and kidney function and inflammatory markers, such as C-reactive protein, were carried out in the Central Laboratory of our hospital.

Since most recent events occurred within 3 months after admission through the review of literature [2] and our clinical experience, we determine that all the included patients were followed up for up to 90 days. The follow-up time was set at 7, 14, 30 and 90 days after admission. All patients were followed up by both telephone interviews and medical record review. The end of the follow-up was the death of all causes. Cause of death was ascertained from the death record, i.e. a legal document including time, site and other information.

Continuous data are expressed as means \pm standard deviation. The unpaired Student's t-test and chi-square test were used for comparisons of continuous and categorical variables, respectively. Kaplan-Meier curves were plotted to evaluate the association between the NLR and the all-cause mortality at follow-up. Univariate and multivariate Cox proportional hazard analysis were used to identify predictors of death. The cut-off point with a maximum combined sensitivity and specificity was selected based on the area under curve (AUC) value from the receiver operator characteristics (ROC) analysis. AP-value of 0.05 or less for the two-sided probability was considered to be statistically significant. All statistical analyses were performed using the SPSS software (SPSS-22.0; IBM Corp., Armonk, NY, USA).

\section{Results}

Baseline characteristic

A total of 341 cases hypertensive patients were enrolled, including 328 males and 13 females, with average age of 
$87.43 \pm 5.21(81-102)$ years. Since the retired army cadres are the main components of the patients for our department, little females were enrolled due to the influence of the restriction. Among the enrolled patients, all patients had been diagnosed with hypertension ranging from 5 to 27 years and had received antihypertensive drug treatment. Additionally, all patients had a history of CAD; 83 patients (24.34\%) had a history of myocardial infarction (MI); 29 patients had received stent therapy. Moreover, 67 cases were with chronic heart failure, while 167 cases with DM. 125 patients were with anemia and all of them received therapy, whose hemoglobin levels in the normal range.

\section{Clinical characteristics according to neutrophil to lymphocyte ratio}

The median and interquartile range of NLR was 3.01 and 2.05-6.12, respectively. All patients were grouped in the quartile based on their NLR score as follows: less than2.05, quartile $1,(n=85)$; between2.05 and2.99, quartile $2(n=88)$; between 3.00 and 6.12 , quartile 3 $(n=83)$; greater than 6.12 , quartile $4(n=85)$. Table 1 showed the baseline characteristics of patients in different groups. Age, male gender, smoking history, history of $\mathrm{MI}$ and stent implantation, chronic heart failure, type 2 DM (T2DM), anemia and chronic kidney disease exhibited no statistical difference among the groups $(p>0.05)$.Hyperlipidemia incidence showed statistically significant difference $(p<0.05)$. Hemodynamic evaluation indicated that patients in the different quartile also had no statistically significant difference in either systolic blood pressure or diastolic blood pressure $(p>0.05)$. The higher quartile was associated with lower serum total cholesterol, triglyceride, serum iron, ejection fraction and platelet distribution width (PDW)as well as higher platelet/lymphocyte ratio, fasting blood glucose (FBG), CRP and white blood cell (WBC) counts $(p<0.05)$.

Enrolled patients were also grouped in the quartile based on their RDW score as follows: less than 12.8, quartile $1,(n=89)$; between 12.8 and 13.4 , quartile 2 $(n=89)$; between 13.4 and 14.2 , quartile $3(n=95)$; greater than 14.2 , quartile $4(n=88)$ and Table 2 showed the baseline characteristics of subjects in different groups. History of chronic heart failure, anemia and chronic kidney disease exhibited statistically significant difference $(p<0.05)$. The higher quartile RDW level was associated with lower serum hemoglobin, ejection fraction $(p<0.05)$. PDW also showed difference among groups but its trend cannot be determined.

\section{Follow-up results}

In total, 27 death were recorded in 90-day follow-up and most took place between the 30 and 90 day $(n=17$,
$62.97 \%)$ after admission. The association between the various quartiles and mortality is presented in Table 3. Patients with the higher quartile are linked to high mortality $(16.47 \%, 13.25 \%, 1.14 \%, 1.17 \%$ respectively; $\left.X^{2}=20.581, P<0.001\right)$. The parameter and characteristics of different outcomes of the patients are shown in Table 4. Surviving patients had a higher BMI $(24.25 \pm 3.05 \mathrm{VS}$ $24.25 \pm 3.05, p=0.012)$ and hemoglobin (123.78 $\pm 17.05 \mathrm{VS}$ $115.07 \pm 20.42, P=0.040)$, as well as lower DBP $(62.48 \pm 9.60$ VS $68.31 \pm 12.02, p=0.016)$, RDW $(13.61 \pm 1.37$ VS $14.18 \pm 1.38, p=0.041)$, platelet lymphocyte ratio (1184.80 \pm 1235.71 VS 1836.84 \pm 1416.97 , $p=0.010)$ and NLR $(4.97 \pm 5.72$ VS $7.95 \pm 6.88, p=0.011)$.

Mapping the ROC of the RDW to predict all-cause mortality (Fig. 1), with an AUC $=0.654(95 \% \mathrm{CI}$ : $0.548-0.761, P=0.008)$, a critical value of $13.2 \%$, a corresponding predictive sensitivity of $81.5 \%$ and a specificity of $43.9 \%$.

The ROC for the NLR is shown in Fig. 2, it had an AUC $=0.714$ (95\%CI: 0.629-0.798, $P<0.001)$, the critical value was 2.97 , with a sensitivity of $92.6 \%$, and a specificity of $52.5 \%$.

The Kaplan-Meier curve showed a significant difference in the all-cause mortality between the different NLR levels (Fig. 3). Admission NLR higher than 2.97 were found to be connected with high mortality compared with those less than 2.97 (1.02\% VS 10.70\%, $p=0.002)$.

The Kaplan-Meier curve of RDW showed there is no significant difference in the all-cause mortality between the different RDW levels $\left(X^{2}=3.680, P=0.055\right.$, Fig. 4$)$.

Univariate and multivariate Cox proportional hazard analyses were performed to investigate the possible predictors of all-cause mortality in the study population (Table 5). It is worth noting that the correlation between the CPR levels and all-cause mortality in such patients is not significant $(\mathrm{RR}=1.042,95 \%$ CI $0.895-1.213$, $P=0.599)$. On the multivariate Cox proportional hazard analysis, using the lowest NLR quartile as reference, 3rd quartiles $(\mathrm{RR}=9.646,95 \% \mathrm{CI} 1.302-34.457, P=0.041)$ and 4th quartiles $(\mathrm{RR}=16.451,95 \%$ CI 2.137-66.643, $P=0.007)$ were found to independently associate with all-cause death in hypertensive patients over 80 years of age, though 2nd quartile $(R R=3.629$, 95\% CI $0.373-18.995, P=0.838$ ) was not. Higher rank of NLR is link to higher incidence of all-cause death for such patients.

Cox proportional hazard analyses were also performed for RDW and the results were shown in Table 6. Increased relative risk was not found among different RDW quartile groups $(\mathrm{RR}=0.737,95 \%$ confidence interval $0.111-4.883, P=0.752$; $R R=1.872$, 95\% CI $0.811-8.252, P=0.088$ and $\mathrm{RR}=2.588,95 \%$ CI $0.096-10.102, P=0.053$, in 2 nd, 3rd, 4th quartile groups respectively). 
Table 1 Baseline characteristics of subjects by the quartile of the neutrophil-lymphocyte ratio

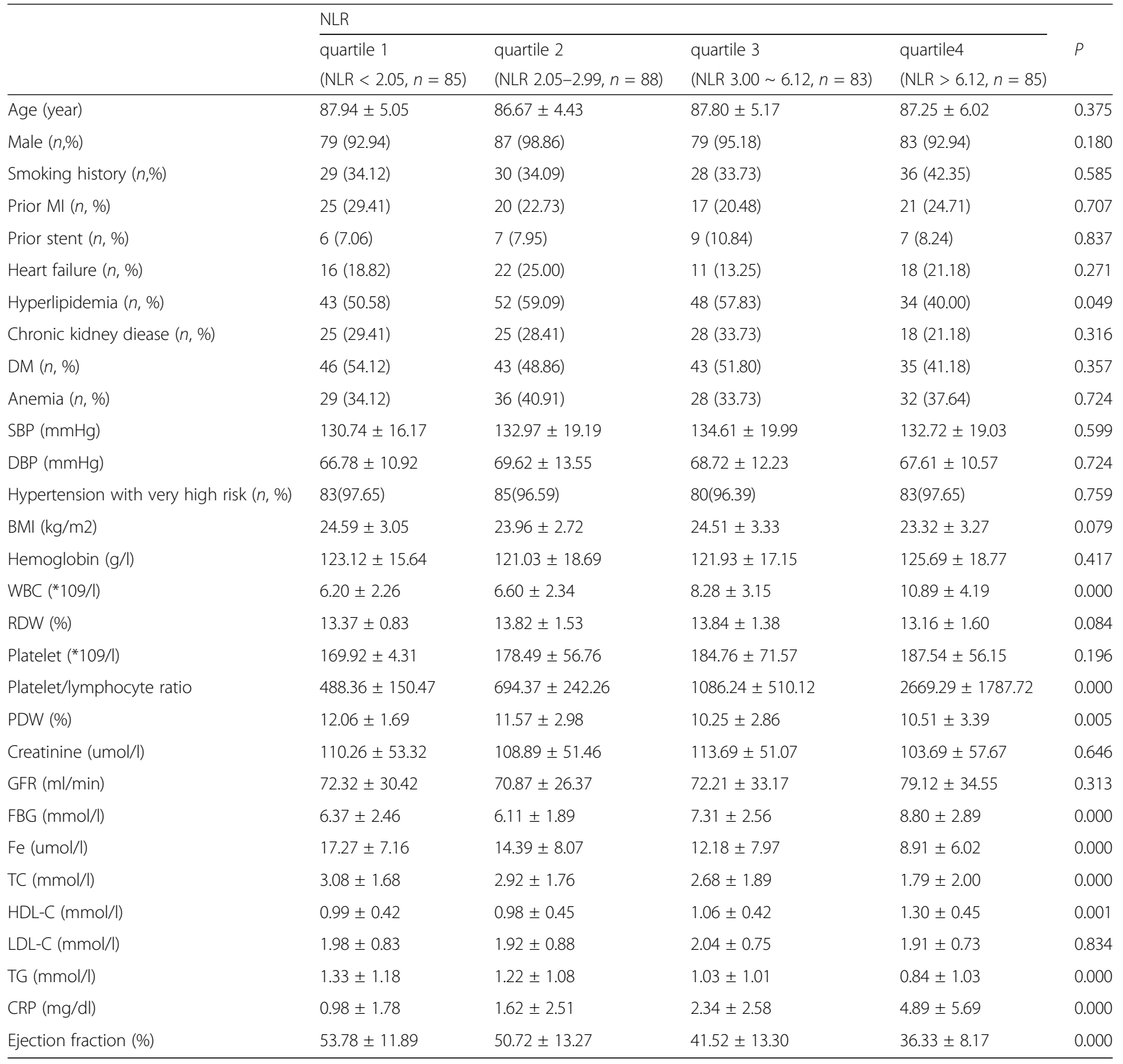

$D M$ Diabetes mellitus, SBP Systolic blood pressure, DBP Diastolic blood pressure, BMI Body mass index, WBC White blood cell, RDW Red cell distribution width, PDW Platelet distribution width, GFR Glomerular filtration rate, FBG Fasting blood glucose, TC Total cholesterol, TG Triglyceride, HDL-C High density lipoprotein cholesterol, LDL-C Low density lipoprotein cholesterol, CRP C-reactive protein

\section{Discussion}

The main finding of the present study was that elevated NLR on admission was an independent predictor of allcause mortality in 90 day for hospitalized hypertensive patients over the age of 80 . With the increase of NLR, the incidence of all-cause death increased (3rd quartiles: $\mathrm{RR}=9.646, P=0.041$ and 4 th quartiles: $\mathrm{RR}=16.451$, $P=0.007)$. Regarding the ROC analysis, an admission NLR higher than 2.97 was found to predict all-cause mortality with a sensitivity of $92.6 \%$ and a specificity of
52.5\% (AUC $=0.714, P=0.00)$. To our knowledge this is the first report of such findings in such patient population.

Inflammation and oxidative stress have been implicated in the pathogenesis of cardiovascular disease, and thus inflammatory biomarkers have received considerable attention. RDW and NLR have recently emerged as potential new biomarkers that discriminate individuals at risk for future adverse events in patients with cardiovascular disease, which were both inexpensive and easily 
Table 2 Baseline characteristics of subjects by the quartile of red cell distribution width

\begin{tabular}{|c|c|c|c|c|c|}
\hline & \multicolumn{4}{|l|}{ RDW } & \multirow{3}{*}{$P$} \\
\hline & quartile 1 & quartile 2 & quartile 3 & quartile4 & \\
\hline & $(\mathrm{RDW}<12.8, n=69)$ & $($ RDW $12.8-13.3, n=89)$ & $($ RDW $13.4 \sim 14.2, n=95)$ & $(\mathrm{RDW}>14.2, n=88)$ & \\
\hline Age (year) & $87.25 \pm 4.76$ & $86.31 \pm 5.43$ & $88.46 \pm 5.25$ & $87.55 \pm 4.92$ & 0.067 \\
\hline Male $(n, \%)$ & 67 (97.10) & $85(95.51)$ & $94(98.95)$ & $82(93.18)$ & 0.210 \\
\hline Smoking history $(n, \%)$ & $23(33.33)$ & $35(39.33)$ & $34(35.79)$ & $31(35.23)$ & 0.882 \\
\hline Prior MI $(n, \%)$ & $20(28.99)$ & $20(22.47)$ & $22(23.15)$ & $24(27.27)$ & 0.166 \\
\hline Prior stent $(n, \%)$ & $7(10.14)$ & $11(12.36)$ & $3(3.15)$ & $8(9.10)$ & 0.099 \\
\hline Heart failure $(n, \%)$ & $7(10.16)$ & $17(19.10)$ & $17(17.89)$ & $26(29.55)$ & 0.022 \\
\hline Hyperlipidemia ( $n, \%)$ & $38(55.07)$ & $39(43.82)$ & $44(46.31)$ & $56(63.64)$ & 0.034 \\
\hline CKD $(n, \%)$ & $14(20.21)$ & $23(25.83)$ & $24(25.26)$ & $36(40.91)$ & 0.021 \\
\hline $\mathrm{DM}(n, \%)$ & $38(55.07)$ & $44(49.43)$ & $47(49.47)$ & $38(43.18)$ & 0.527 \\
\hline Anemia $(n, \%)$ & $15(21.73)$ & $26(29.21)$ & $39(41.05)$ & $45(51.10)$ & 0.011 \\
\hline $\mathrm{SBP}(\mathrm{mmHg})$ & $132.52 \pm 18.27$ & $132.17 \pm 18.43$ & $131.28 \pm 19.04$ & $135.21 \pm 18.72$ & 0.529 \\
\hline $\mathrm{DBP}(\mathrm{mmHg})$ & $70.56 \pm 15.08$ & $61.62 \pm 10.51$ & $68.52 \pm 11.19$ & $65.71 \pm 10.95$ & 0.062 \\
\hline Hypertension with very high risk $(n, \%)$ & $67(97.10)$ & $85(95.50)$ & $92(96.84)$ & 87 (98.86) & 0.778 \\
\hline BMI (kg/m2) & $24.73 \pm 3.17$ & $23.77 \pm 2.86$ & $23.91 \pm 3.21$ & $24.26 \pm 3.15$ & 0.300 \\
\hline Hemoglobin (g/l) & $128.79 \pm 13.67$ & $125.04 \pm 16.80$ & $122.29 \pm 16.87$ & $117.52 \pm 19.77$ & 0.010 \\
\hline WBC $\left({ }^{*} 109 / 1\right)$ & $7.67 \pm 3.78$ & $7.37 \pm 3.21$ & $8.15 \pm 3.53$ & $8.75 \pm 3.75$ & 0.061 \\
\hline Platelet $\left({ }^{*} 109 / 1\right)$ & $173.83 \pm 49.55$ & $179.10 \pm 52.42$ & $180.41 \pm 52.30$ & $186.13 \pm 72.84$ & 0.612 \\
\hline PDW (\%) & $10.60 \pm 3.42$ & $11.27 \pm 2.48$ & $11.88 \pm 1.73$ & $11.43 \pm 1.63$ & 0.049 \\
\hline Creatinine (umol/l) & $96.29 \pm 28.91$ & $102.37 \pm 34.73$ & $109.86 \pm 34.74$ & $125.03 \pm 72.26$ & 0.040 \\
\hline GFR (ml/min) & $78.48 \pm 29.16$ & $74.02 \pm 26.46$ & $72.51 \pm 32.65$ & $68.50 \pm 35.67$ & 0.252 \\
\hline $\mathrm{FBG}(\mathrm{mmol} / \mathrm{l})$ & $7.26 \pm 2.83$ & $6.83 \pm 2.51$ & $6.98 \pm 2.50$ & $7.01 \pm 2.57$ & 0.787 \\
\hline Fe (umol/l) & $13.73 \pm 8.23$ & $15.09 \pm 9.53$ & $12.15 \pm 6.06$ & $11.91 \pm 7.41$ & 0.029 \\
\hline TC $(\mathrm{mmol} / \mathrm{l})$ & $2.87 \pm 2.00$ & $2.83 \pm 1.78$ & $2.50 \pm 1.97$ & $2.53 \pm 1.87$ & 0.621 \\
\hline $\mathrm{HDL}-\mathrm{C}(\mathrm{mmol} / \mathrm{l})$ & $1.05 \pm 0.41$ & $1.14 \pm 0.43$ & $1.01 \pm 0.48$ & $1.04 \pm 0.45$ & 0.319 \\
\hline LDL-C (mmol/l) & $2.14 \pm 0.97$ & $1.97 \pm 0.64$ & $1.91 \pm 0.95$ & $1.92 \pm 0.62$ & 0.387 \\
\hline TG $(\mathrm{mmol} / \mathrm{l})$ & $1.08 \pm 1.18$ & $0.99 \pm 0.90$ & $0.91 \pm 1.00$ & $1.16 \pm 136$ & 0.477 \\
\hline CRP (mg/dl) & $2.33 \pm 4.75$ & $2.80 \pm 3.18$ & $2.78 \pm 4.30$ & $2.85 \pm 3.71$ & 0.900 \\
\hline Ejection fraction(\%) & $48.70 \pm 13.61$ & $46.61 \pm 13.73$ & $44.93 \pm 14.25$ & $42.29 \pm 12.30$ & 0.023 \\
\hline
\end{tabular}

RDW Red cell distribution width, DM Diabetes mellitus, CKD Chronic kidney disease, SBP Systolic blood pressure, DBP Diastolic blood pressure, BMI Body mass index, WBC White blood cell, RDW Red cell distribution width, PDW Platelet distribution width, GFR Glomerular filtration rate, FBG Fasting blood glucose, TC Total cholesterol, TG Triglyceride, HDL-C High density lipoprotein cholesterol, LDL-C Low density lipoprotein cholesterol, CRP C-reactive protein

accessible $[8,9]$. Simple indicators that can provide a wealth of information for clinical hypertension deserve more attention.

Indeed, increased RDW was found to be an independent predictor of mortality in patients with heart failure

Table 3 All-cause death in different quartile

\begin{tabular}{lllll}
\hline Group & \multicolumn{4}{l}{ All-cause death } \\
\cline { 2 - 5 } & Day 7 & Day 14 & Day 30 & Day 90 \\
\hline quartile1 $(n, \%)$ & $1(1.18)$ & $0(0.00)$ & $0(0.00)$ & $0(0.00)$ \\
quartile2 $(n, \%)$ & $0(0.00)$ & $0(0.00)$ & $1(1.14)$ & $0(0.00)$ \\
quartile3 $(n, \%)$ & $0(0.00)$ & $1(1.14)$ & $2(2.41)$ & $8(9.41)$ \\
quartile4 $(n, \%)$ & $2(2.35)$ & $1(1.14)$ & $2(2.41)$ & $9(10.59)$ \\
\hline
\end{tabular}

[10], further studies showed that the RDW is a prognostic indicator for patients with HF caused by coronary heart disease and dilated cardiomyopathy [11]. The RDW is also an independent predictor of the coronary artery calcification, suggesting that it might be a useful marker for predicting CAD [12]. Elevated RDW levels, may be an independent risk marker for non-valvular $\mathrm{AF}$ [13]. Accordingly, the RDW is considered to be a good indicator of the prognosis of cardiovascular diseases, but few studies have been carried out in hypertensive patients. Sarikaya et al. [14] found that the RDW levels were higher in hypertensive patients with AF. An increased RDW level in such patients with hypertension may alert physician on the development or presence of 
Table 4 Comparison of the laboratory parameters of different outcome

\begin{tabular}{llll}
\hline & $\begin{array}{l}\text { Death for all cause } \\
(n=27)\end{array}$ & $\begin{array}{l}\text { Survival } \\
(n=314)\end{array}$ & $P$ \\
\hline Age (year) & $89.29 \pm 4.57$ & $87.26 \pm 5.25$ & 0.052 \\
Prior MI $(n, \%)$ & $7(25.93)$ & $79(25.16)$ & 0.943 \\
Heart failure $(n, \%)$ & $3(11.11)$ & $64(20.38)$ & 0.318 \\
DM (n,\%) & $15(55.56)$ & $152(48.41)$ & 0.304 \\
Hyperlipidemia $(n, \%)$ & $12(44.44)$ & $165(52.55)$ & 0.431 \\
BMI (kg/m $\left.{ }^{2}\right)$ & $22.31 \pm 3.31$ & $24.25 \pm 3.05$ & 0.012 \\
SBP(mmHg, & $133.29 \pm 18.43$ & $126.85 \pm 20.16$ & 0.085 \\
DBP (mmHg) & $68.31 \pm 12.02$ & $62.48 \pm 9.60$ & 0.016 \\
Hemoglobin (g/l) & $115.07 \pm 20.42$ & $123.78 \pm 17.05$ & 0.040 \\
WBC (*10 $/$ l) & $8.90 \pm 5.62$ & $7.92 \pm 3.35$ & 0.176 \\
RDW (\%) & $14.18 \pm 1.38$ & $13.61 \pm 1.37$ & 0.041 \\
Platelet $\left({ }^{*} 10^{9} / l\right)$ & $188.07 \pm 60.65$ & $179.55 \pm 57.54$ & 0.463 \\
Platelet/lymphocyte ratio & $1836.84 \pm 1416.97$ & $1184.80 \pm 1235.71$ & 0.010 \\
NLR & $7.95 \pm 6.88$ & $4.97 \pm 5.72$ & 0.011 \\
Creatinine (umol/l) & $110.74 \pm 61.19$ & $108.97 \pm 52.44$ & 0.868 \\
CRP (mg/dl) & $3.49 \pm 2.88$ & $2.63 \pm 4.08$ & 0.316 \\
\hline
\end{tabular}

MI Myocardial infarction, DM Diabetes mellitus, SBP Systolic blood pressure, $D B P$ Diastolic blood pressure, $B M I$ Body mass index, RDW Red cell distribution width, CRP C-reactive protein

AF. Tanindi et al. [15] found that higher RDW values are strongly correlated with higher systolic and diastolic blood pressures. But in our study revealed that, though the RDW level showed difference between surviving and death, Cox proportional hazard analyses indicated higher

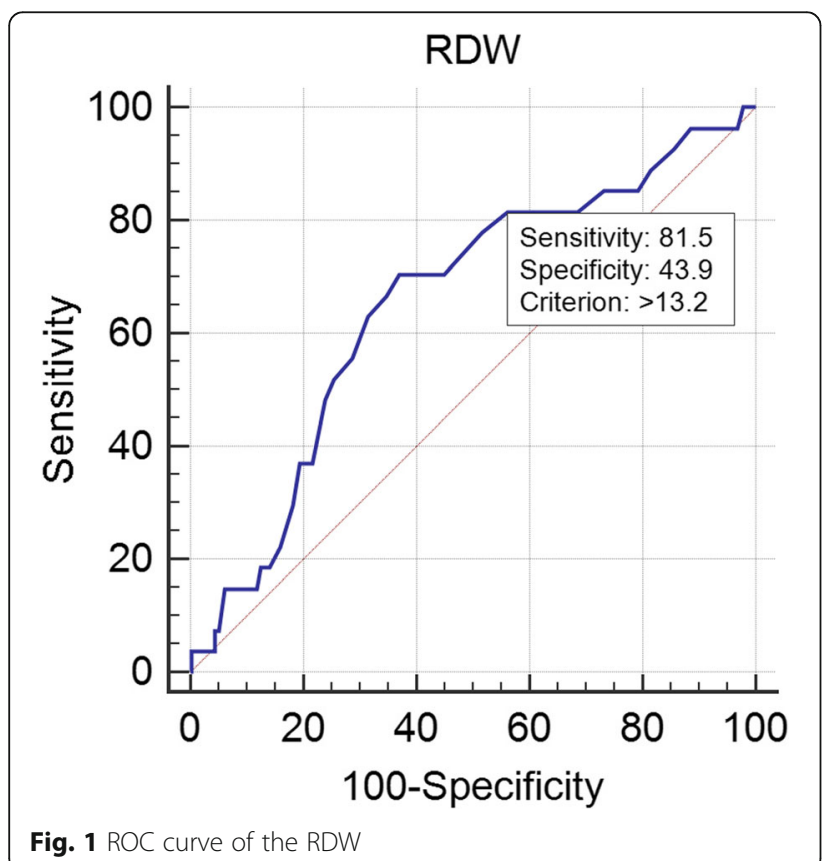

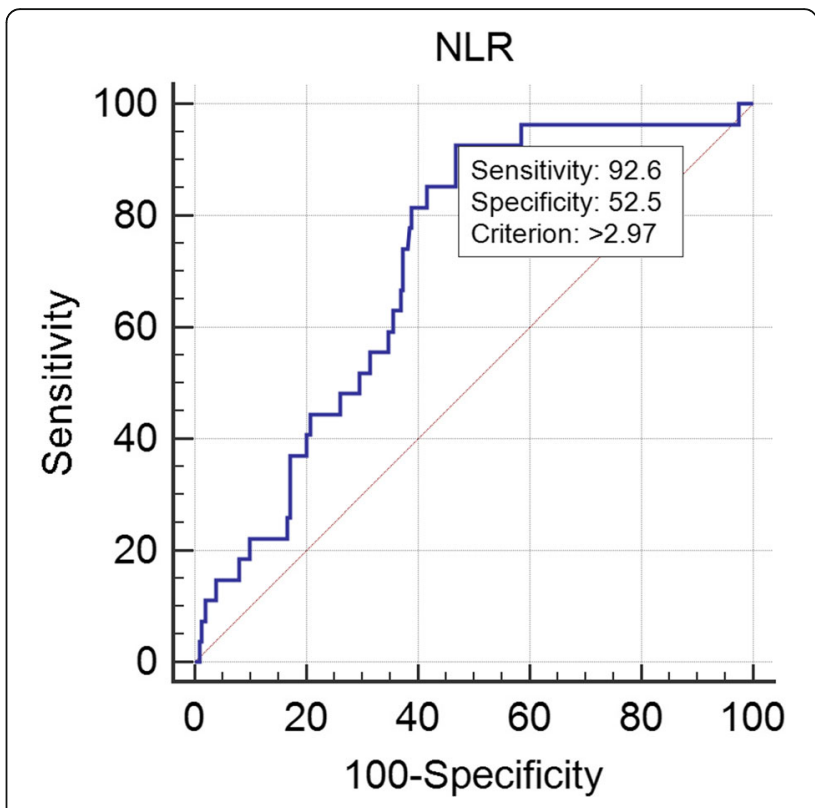

Fig. 2 ROC curve of the NLR

RDW level wasn't accompanied with an increased risk of all-cause mortality 90 days after admission.

Unlike many other inflammatory markers and bioassays, the NLR is an inexpensive and readily available marker which is a combination of two independent markers of inflammation, providing us with additional information. It also can be an index for of sympathetic/ parasympathetic tone balance. A higher level of NLR could indicate a higher ratio of sympathetic/parasympathetic tone [16]. So it may provide us with more information in cardiovascular disease. Recently, many studies focused on the NLR and their association with adverse outcomes in patients with cardiovascular disease, but most of these studies focused on CAD or heart failure, especially in patients with ACS. High NLR levels, white blood cell counts, and neutrophil counts at admission are independently correlated with stent restenosis after primary PCI $[17,18]$. In ST elevation myocardial infarction patients, the frequency of ventricular tachyarrhythmia (VT/VF) at the first day was associated with higher neutrophil count $(P<0.001)$ and higher NLR level $(P<0.001)$ [19]. Average NLR was a useful and powerful predictor of mortality and adverse-outcomes in Chinese patients presenting with ST segment elevation myocardial infarction [20]. Benites et al. [21] found intermediate and high NLR tertiles remained significantly associated with all-cause mortality ( $\mathrm{HR}=1.83,95 \% \mathrm{CI} 1.07$ to 3.14 and $\mathrm{HR}=2.16,95 \% \mathrm{CI} 1.21$ to 3.83 ) in advanced heart failure. Cut-off value of 5.1 for NLR could predict death in HF patients with $75 \%$ sensitivity and $62 \%$ specificity during a 12.8-month follow-up period on average, thus 


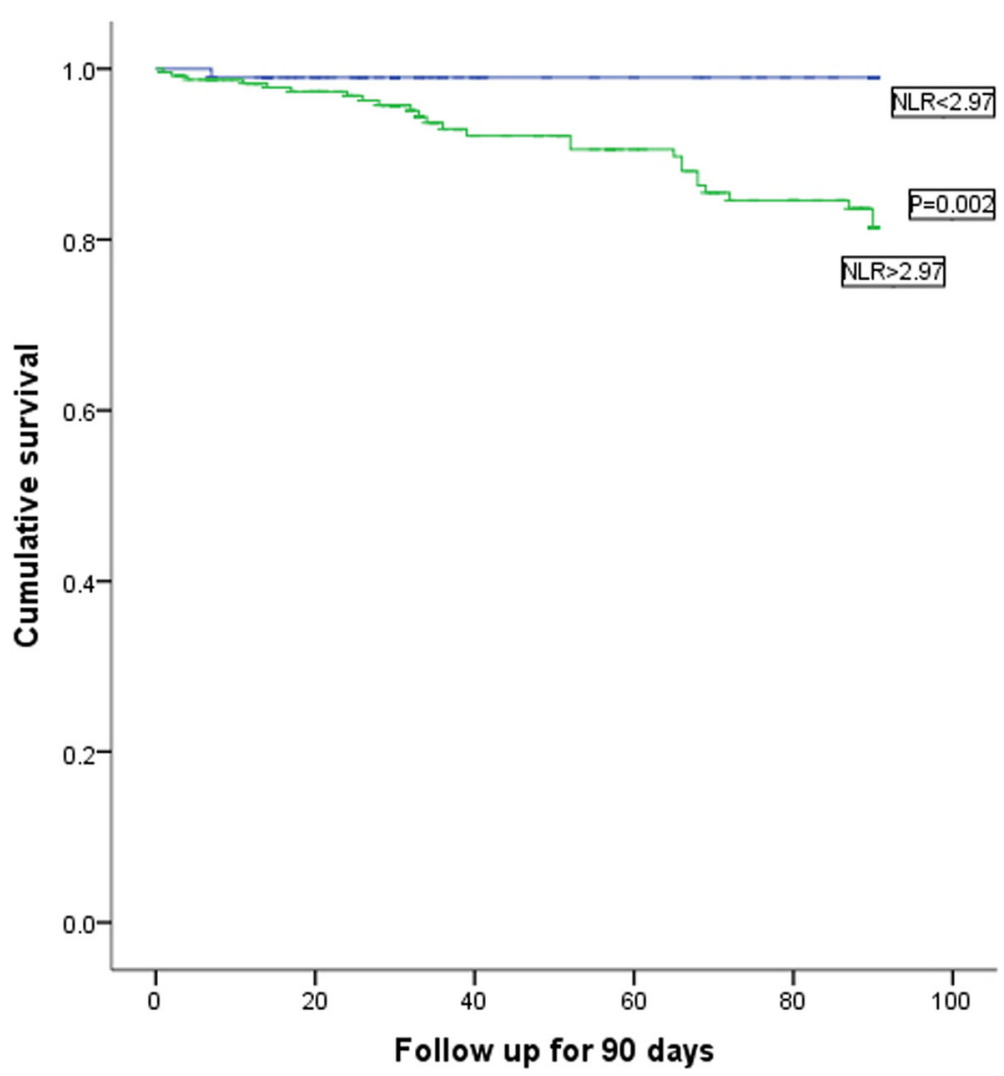

Fig. 3 The Kaplan-Meier survival curves for different NLR levels

NLR was considered to be used to predict mortality during the follow-up of HF patients [22].

Fewer studies have focused on NLR and hypertension. A study on resistance hypertension showed that NLR level was increased in hypertension patients and the

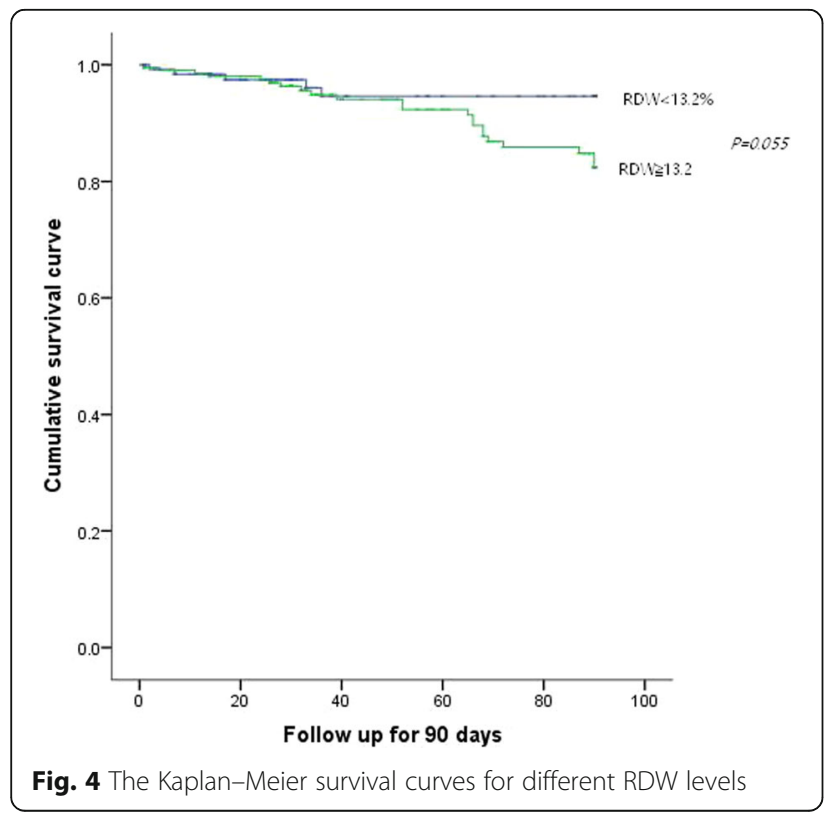

resistance hypertension group had a significantly higher NLR than the control hypertension group $(P=0.03)$ [23]. Hypertensive patients with high homocysteine(HCY) had increased NLR and it positively correlated with HCY but not with blood pressure [24]. Also, there was a statistically significant positive correlation between the ascending aortic diameter and NLR $(r=0.524$, $P<0.001$ ), which indicated that it plays a role in the pathogenesis of aneurysm of the ascending aorta in hypertensive patients [25]. In addition, hypertensive

Table 5 Multivariate Cox hazard model NLR for the possible predictors of all-cause death in the study population

\begin{tabular}{lllll}
\hline & HR & P & IC.inf & IC.sup \\
\hline Quartile 2 & 3.629 & 0.838 & 0.377 & 18.995 \\
Quartile 3 & 9.646 & 0.041 & 1.302 & 34.457 \\
Quartile 4 & 16.451 & 0.007 & 2.137 & 66.643 \\
SBP & 0.993 & 0.678 & 0.962 & 1.025 \\
DBP & 0.956 & 0.096 & 0.906 & 1.008 \\
BMI & 0.898 & 0.190 & 0.765 & 1.055 \\
Age & 1.126 & 0.053 & 0.998 & 1.271 \\
CRP & 1.042 & 0.599 & 0.895 & 1.213 \\
\hline RDW Red cell distribution width, SBP Systolic blood pressure, DBP Diastolic \\
blood pressure, BMI Body mass index, CRP C-reactive protein, HR hazard ratio.
\end{tabular}


Table 6 Multivariate Cox hazard model of RDW for the possible predictors of All-cause death in the study population

\begin{tabular}{lllll}
\hline & HR & $P$ & IC.inf & IC.sup \\
\hline RDW Quartile 2 & 0.737 & 0.752 & 0.111 & 4.883 \\
RDW Quartile 3 & 1.872 & 0.088 & 0.811 & 8.252 \\
RDW Quartile 4 & 2.588 & 0.053 & 0.960 & 10.012 \\
SBP & 0.982 & 0.315 & 0.949 & 1.017 \\
DBP & 0.972 & 0.330 & 0.918 & 1.029 \\
BMI & 0.901 & 0.266 & 0.750 & 1.083 \\
Age & 1.094 & 0.163 & 0.965 & 1.240 \\
CRP & 1.113 & 0.117 & 0.973 & 1.273 \\
\hline RDW Red
\end{tabular}

$R D W$ Red cell distribution width, SBP Systolic blood pressure, $D B P$ Diastolic blood pressure, BMI Body mass index, CRP C-reactive protein, HR hazard ratio

patients with diastolic dysfunction had higher values of NLR compared with subjects without diastolic dysfunction. Furthermore higher grades of diastolic dysfunction were associated with higher levels of NLR [26]. And patients with non-dipper hypertension had significantly higher NLR and PLR compared to dipper hypertension, which has not been reported previously [27]. All these findings suggest that higher NLR values may link to poor clinical outcome in subtypes of hypertension, which make us believed that NLR could be used for risk stratification and it may be a good predictor for the prognosis of hypertension. Now our research first confirms such points. We found that a higher quartile of NLR was tend to have a higher incidence of death, and increased NLR quartile will increased the risk of all-cause death (3rd quartiles: $\mathrm{RR}=9.646,95 \%$ confidence interval 1.30234.457, $P=0.041$ and 4th quartiles: $R R=16.451,95 \%$ confidence interval 2.137-66.643, $P=0.007$ ). So we believed that high NLR level was an independent predictor of all-cause mortality in hypertensive patients over 80 years of age, which provided us convenient and preliminary screening tool.

NLR can be easily calculated from complete blood cell count performed in nearly every patient. With this knowledge, NLR may lead physicians to identify high risk patients who require closer care because of increased risks of all-cause death easily. And will help us to use the medical resources more efficient. Together with other index, the specificity may improve. Our results may provide a simple and effective tool of preliminary screening for such kind of patients, which is the most important value of research. We also see that NLR have high sensitivity but low specificity, which hint us that high NLR level is just a preliminary tool and it may be necessary to combine with other indicators to provide more accurate evaluation information.

The limitations of the present study are as follows. (1) This was a single center study that included a relatively small number of patients. (2) Only one measurement of admission full blood count and calculation of RDW and NLR was included in the analysis.

\section{Conclusion}

We found that higher NLR levels in hypertensive patients admitted to the hospital are good predictors for all-cause mortality 90 days after admission. This indicated that NLR, which is easily determinable, broadly available and inexpensive markers, could be used to identify patients at high risk for adverse endpoints. However, these findings must be confirmed on a study with a larger patient population.

\section{Abbreviations \\ ACS: Acute coronary syndrome; AF: Atrial fibrillation; AUC: Area under curve; BMI: Body mass index; CAD: Coronary artery disease; CRP: C-reactive protein; DBP: Diastolic blood pressure; DM: Diabetes mellitus; FBG: Fasting blood glucose; GFR: Glomerular filtration rate; HCY: Homocysteine; HDL-C: High density lipoprotein cholesterol; HF: Heart failure; Hgb: Hemoglobin; LDL-C: Low density lipoprotein cholesterol; NLR: Neutrophil-to-lymphocyte ratio; PAD: Peripheral artery disease; PDW: Platelet distribution width; RDW: Red cell distribution width; ROC: Receiver operator characteristics; SBP: Systolic blood pressure; SLE: Systemic lupus erythematosus; TC: Total cholesterol; TG: Triglyceride}

\section{Acknowledgements}

I would like to extend my sincere gratitude to Dr. Jin Zheng, Yuan Liu, for their contribution and support in the clinical data collection work.

\section{Funding}

This study is funded by a grant from the Chinese PLA Health Project (Project ID: 12BJZ34).

Availability of data and materials

All the data and material are available.

Authors' contributions

SXN, LLM and YP contributed to the design of the review. SXN, ZXQ, DRX acquired the data. SXN, LLM and ZXQ contributed to analysis. SXN wrote the draft. SXN, LLM, ZXQ, YP and DRX critically revised the intellectual content of this work.

Competing interests

The authors declare that they have no competing of interests.

Consent for publication

We give our consent for this material to publish in BMC Cardiovascular Disorders.

Ethics approval and consent to participate

The current study was conducted at Chinese PLA general hospitals with full ethical approval of the Human Investigation Committee. In addition, informed consent was obtained from all patients.

\section{Publisher's Note}

Springer Nature remains neutral with regard to jurisdictional claims in published maps and institutional affiliations.

\section{Author details}

'Department of Geriatric Cardiology, Chinese People's Liberation Army General Hospital, 28 Fuxing Road, Beijing 100853, People's Republic of China. ²Department of Cardiology, Chinese People's Liberation Army 305 Hospital, Beijing 100000, China. 
Received: 24 December 2016 Accepted: 7 June 2017

Published online: 24 June 2017

\section{References}

1. Fowler C. CRP \& cardiovascular disease. Measuring C-reactive protein can help assess a patient's CVD risk. Advance for NPs \& PAs. 2011;2(12):23-7. quiz 28

2. Bhat T, Teli S, Rijal J, Bhat H, Raza M, Khoueiry G, et al. Neutrophil to lymphocyte ratio and cardiovascular diseases: a review. Expert Rev Cardiovasc Ther. 2013;11(1):55-9.

3. Arbel $Y$, Shacham $Y$, Finkelstein A, Halkin A, Milwidsky A, Berliner $S$, et al. Red blood cell distribution width (RDW) and long-term survival in patients with ST elevation myocardial infarction. Thromb Res. 2014;134(5):976-9.

4. Wu Y, Chen Y, Yang X, Chen L, Yang Y. Neutrophil-to-lymphocyte ratio (NLR) and platelet-to-lymphocyte ratio (PLR) were associated with disease activity in patients with systemic lupus erythematosus. Int Immunopharmacol. 2016;36:94-9.

5. Marchioni M, Primiceri G, Ingrosso M, Filograna R, Castellan P, De Francesco $P$, et al. The clinical use of the neutrophil to lymphocyte ratio (NLR) in Urothelial cancer: a systematic review. Clin Genitourin Cancer. 2016;

6. Danese E, Lippi G, Montagnana M. Red blood cell distribution width and cardiovascular diseases. Journal of thoracic disease. 2015;7(10):E402-11.

7. Liu LS. Writing Group of Chinese Guidelines for the Management of $\mathrm{H}$ : [2010 Chinese guidelines for the management of hypertension]. Zhonghua xin xue guan bing za zhi. 2011;39(7):579-615.

8. Uyarel H, Isik T, Ayhan E, Ergelen M. Red cell distrubition width (RDW): a novel risk factor for cardiovascular disease. Int J Cardiol. 2012;154(3):351-2.

9. Afari ME, Bhat T. Neutrophil to lymphocyte ratio (NLR) and cardiovascular diseases: an update. Expert Rev Cardiovasc Ther. 2016;14(5):573-7.

10. Allen LA, Felker GM, Mehra MR, Chiong JR, Dunlap SH, Ghali JK, et al. Validation and potential mechanisms of red cell distribution width as a prognostic marker in heart failure. J Card Fail. 2010;16(3):230-8.

11. Zhang Y, Wang Y, Kang JS, Yu JX, Yin SJ, Cong XF, et al. Differences in the predictive value of red cell distribution width for the mortality of patients with heart failure due to various heart diseases. Journal of geriatric cardiology : JGC. 2015;12(6):647-54

12. Gurel OM, Demircelik MB, Bilgic MA, Yilmaz H, Yilmaz OC, Cakmak M, et al. Association between red blood cell distribution width and coronary artery calcification in patients undergoing 64-Multidetector computed tomography. Korean circulation journal. 2015;45(5):372-7.

13. Gungor B, Ozcan KS, Erdinler I, Ekmekci A, Alper AT, Osmonov D, et al. Elevated levels of RDW is associated with non-valvular atrial fibrillation. $J$ Thromb Thrombolysis. 2014;37(4):404-10.

14. Sarikaya S, Sahin S, Akyol L, Borekci E, Yilmaz YK, Altunkas F, et al. Is there any relationship between RDW levels and atrial fibrillation in hypertensive patient? Afr Health Sci. 2014;14(1):267-72.

15. Tanindi A, Topal FE, Topal F, Celik B. Red cell distribution width in patients with prehypertension and hypertension. Blood Press. 2012;21(3):177-81.

16. Abo T, Kawamura T. Immunomodulation by the autonomic nervous system: therapeutic approach for cancer, collagen diseases, and inflammatory bowel diseases. Therapeutic apheresis : official journal of the International Society for Apheresis and the Japanese Society for Apheresis. 2002;6(5):348-57.

17. Bolca O, Gungor B, Ozcan KS, Karadeniz FO, Sungur A, Koroglu B, et al. The neutrophil-to-lymphocyte ratio is associated with bare-metal stent restenosis in STEMI patients treated with primary $\mathrm{PCl}$. Coron Artery Dis. 2015;26(5):402-8.

18. Fowler AJ, Agha RA. Neutrophil/lymphocyte ratio is related to the severity of coronary artery disease and clinical outcome in patients undergoing angiography-the growing versatility of NLR. Atherosclerosis. 2013;228(1):44-5.

19. Ghaffari S, Nadiri M, Pourafkari L, Sepehrvand N, Movasagpoor A, Rahmatvand $\mathrm{N}$, et al. The predictive value of Total neutrophil count and neutrophil/lymphocyte ratio in predicting in-hospital mortality and complications after STEMI. J Cardiovasc Thorac Res. 2014;6(1):35-41.

20. He J, Li J, Wang Y, Hao P, Hua Q. Neutrophil-to-lymphocyte ratio (NLR) predicts mortality and adverse-outcomes after ST-segment elevation myocardial infarction in Chinese people. Int J Clin Exp Pathol. 2014;7(7):4045-56

21. Benites-Zapata VA, Hernandez AV, Nagarajan V, Cauthen CA, Starling RC, Tang WH. Usefulness of neutrophil-to-lymphocyte ratio in risk stratification of patients with advanced heart failure. Am J Cardiol. 2015;115(1):57-61.
22. Durmus E, Kivrak T, Gerin F, Sunbul M, Sari I, Erdogan O. Neutrophil-tolymphocyte ratio and platelet-to-lymphocyte ratio are predictors of heart failure. Arq Bras Cardiol. 2015;105(6):606-13.

23. Belen E, Sungur A, Sungur MA, Erdogan G. Increased neutrophil to lymphocyte ratio in patients with resistant hypertension. J Clin Hypertens (Greenwich). 2015;17(7):532-7.

24. Gang L, Yanyan Z. Increased neutrophil to lymphocyte ratio in persons suffering from hypertension with hyperhomocysteinemia. Hypertens Res. 2016;39(8):606-11.

25. Cem O, Yilmaz S, Korkmaz A, Fahrettin T, Sahin I, Demir V. Evaluation of the neutrophil-lymphocyte ratio in newly diagnosed nondiabetic hypertensive patients with ascending aortic dilatation. Blood Press Monit. 2016;21(4):238-43.

26. Karagoz A, Vural A, Gunaydin ZY, Bektas O, Gul M, Celik A, et al. The role of neutrophil to lymphocyte ratio as a predictor of diastolic dysfunction in hypertensive patients. Eur Rev Med Pharmacol Sci. 2015:19(3):433-40.

27. Sunbul M, Gerin F, Durmus E, Kivrak T, Sari I, Tigen K, et al. Neutrophil to lymphocyte and platelet to lymphocyte ratio in patients with dipper versus non-dipper hypertension. Clin Exp Hypertens. 2014;36(4):217-21.

\section{Submit your next manuscript to BioMed Central and we will help you at every step:}

- We accept pre-submission inquiries

- Our selector tool helps you to find the most relevant journal

- We provide round the clock customer support

- Convenient online submission

- Thorough peer review

- Inclusion in PubMed and all major indexing services

- Maximum visibility for your research

Submit your manuscript at www.biomedcentral.com/submit
C) Biomed Central 